\title{
PLD2 regulates microtubule stability and spindle migration in mouse oocytes during meiotic division
}

\author{
Xiaoyu Liu ${ }^{1}$, Xiaoyun Liu ${ }^{1}$, Dandan Chen ${ }^{1}$, Xiuying Jiang ${ }^{1}$, Wei Ma ${ }^{\text {Corresp. }}{ }^{1}$ \\ ${ }^{1}$ Department of Histology and Embryology, School of Basic Medical Sciences, Capital Medical University, Beijing, China \\ Corresponding Author: Wei Ma \\ Email address: mawei1026@ccmu.edu.cn
}

Phospholipase D2 (PLD2) is involved in cytoskeletal reorganization, cell migration, cell cycle progression, transcriptional control and vesicle trafficking. There is no evidence about PLD2 function in oocytes during meiosis. Herein, we analyzed PLD2 expression and its relationship with spindle formation and positioning in mouse oocyte meiosis. High protein level of PLD2 was revealed in oocytes by western blot, which remained consistently stable from prophase I with intact germinal vesicle (GV) up to metaphase II (MII) stage. Immunofluorescence showed that PLD2appeared and gathered around the condensed chromosomesafter germinal vesicle breakdown (GVBD), and co-localizedwith spindle from pro-metaphase I (pro-MI) to metaphase I (MI) and at MII stage. During anaphase I (Ana I) to telophase I (Tel I) transition, PLD2 was concentrated in spindle polar area but absent from midbody. In oocytes incubated with NFOT, an allosteric and catalytic inhibitor to PLD2, the spindle was enlarged and center-positioned, microtubules were resistant to cold-induced depolymerization, and additionally, the meiotic progression was arrested at MI stage. However, spindle migration could not be totally prevented by PLD2 catalytic specific inhibitors, FIPI and 1-butanol, implying at least partially, that PLD2 effect on spindle migration needs non-catalytic domain participation. NFOT-induced defects also resulted in actin-related molecules' distribution alteration, such as RhoA, phosphatidylinosital 4, 5biphosphate (PIP2), phosphorylated Colifin, and consequently, unordered F-actin dynamics. Taken together, these data indicate PLD2 is required for the regulation ofmicrotubule dynamics and spindle migration toward the cortex in mammalian oocytes during meiotic progression. 
$1 \quad$ PLD2 regulates microtubule stability and spindle migration in mouse oocytes during

2 meiotic division

3

4 Xiaoyu Liu ${ }^{1}$, Xiaoyun $\mathrm{Liu}^{1}$, Dandan $\mathrm{Chen}^{1}$, Xiuying Jiang ${ }^{1}$, Wei Ma ${ }^{1,2}$

$5{ }^{1}$ Department of Histology and Embryology, School of Basic Medical Sciences, Capital Medical

6 University, Beijing, 100069, China

7

8 Running title: PLD2 requirement in spindle positioning

9

$10 \quad{ }^{2}$ Correspondence

11 Wei Ma, E-mail: mawei1026@ccmu.edu.cn. 


\section{ABSTRACT}

13 Phospholipase D2 (PLD2) is involved in cytoskeletal reorganization, cell migration, cell cycle progression, transcriptional control and vesicle trafficking. There is no evidence about PLD2

15 function in oocytes during meiosis. Herein, we analyzed PLD2 expression and its relationship with spindle formation and positioning in mouse oocyte meiosis. High protein level of PLD2 was revealed in oocytes by western blot, which remained consistently stable from prophase I with intact germinal vesicle (GV) up to metaphase II (MII) stage. Immunofluorescence showed that PLD2 appeared and gathered around the condensed chromosomes after germinal vesicle breakdown (GVBD), and co-localized with spindle from pro-metaphase I (pro-MI) to metaphase I (MI) and at MII stage. During anaphase I (Ana I) to telophase I (Tel I) transition, PLD2 was concentrated in spindle polar area but absent from midbody. In oocytes incubated with NFOT, an allosteric and catalytic inhibitor to PLD2, the spindle was enlarged and center-positioned, microtubules were resistant to cold-induced depolymerization, and additionally, the meiotic progression was arrested at MI stage. However, spindle migration could not be totally prevented by PLD2 catalytic specific inhibitors, FIPI and 1-butanol, implying at least partially, that PLD2 effect on spindle migration needs non-catalytic domain participation. NFOT-induced defects also resulted in actin-related molecules' distribution alteration, such as RhoA, phosphatidylinosital 4, 5- biphosphate (PIP2), phosphorylated Colifin, and consequently, unordered F-actin dynamics. Taken together, these data indicate PLD2 is required for the regulation of microtubule dynamics and spindle migration toward the cortex in mammalian oocytes during meiotic progression. 


\section{INTRODUCTION}

Meiotic maturation of oocyte is characterized by two rounds of accurate segregations of genome and asymmetric divisions of cytoplasm, resulting in two tiny polar bodies and one large oocyte. The asymmetric division is critical for the oocyte to store as much material stores as possible for the latter embryo development (Maro \& Verlhac, 2002). The asymmetric division firstly depends on the proper location of spindle (Gonczy et al., 2002). In mitotic animal cells, astral microtubules interact with the cortex to position the cleavage furrow, however, in meiotic oocytes, which are absent of centrioles and astral microtubules (Almonacid \& Paoletti, 2010; von Dassow et al., 2009), a substitution mechanism is established to locate the spindle position. In mouse oocyte, spindle migration is an actin-dependent event rather than a microtubule-associated mechanism. Microtubule depolymerization with nocodazole treatment did not affect the faster cortex-towards movement of chromosomes ( $\mathrm{Li}$ et al., 2008), however, either the stabilization of actin polymerization by jasplakinolide or its depolymerization by cytochalasin B, all impeded the migration of spindle and chromosomes group to the cortex (Sathananthan et al., 2006; Li et al., 2008).

The actin meshwork in consistent remodeling state is needed in order to 'move' the spindle, which has been ascertained by the visualization of actin with developed probes in oocytes alive. The actin network density is initially high at prophase I, then dropped shortly around germinal vesicle breakdown (GVBD), but gradually increased as spindle migration to cortex during meiosis I progression (Chaigne et al., 2012). Actin dynamic is regulated by actin nucleators, including Formin 2 (FMN2) and Arp2/3 complex. FMN2 is a 'straight' actin nucleator, in accompany with spire $1 / 2$, mainly involved in actin nucleation in cytoplasm area, and the 'branched' actin nucleater Arp2/3 dominantly regulates actin nucleation in the cortex. In addition, 
58 there are also other factors regulating actin polymerization or depolymerization, including small

59 GTPase Rho A, phosphatidylinosital 4, 5- biphosphate (PIP2) and Colifin. It is known that Rho

60 A regulates the reorganization of filament actin (F-actin). The reduction of PIP2 synthesis

61 suppresses actin polymerization and motility, while increasing PIP2 synthesis promotes these

62 activities. Colifin, known as ADF/Colifin, which splices F-actin into small pieces, will lose its

63 splicing activity after being phosphorylated at Ser3 by several proteins, such as LIM kinase 1

64 (LIMK1), so the cellular level of p-Colifin ${ }^{\mathrm{Ser} 3}$ could indirectly show the actin assemble state

65 (Pfender et al., 2011; Sun et al., 2011).

66 Phospholipase D2 (PLD2), one phospholipase D isoform, contains two conserved HKD

67 domains in C-terminus, one phox homology (PX) and one pleckstrin homology (PH) domain in

68 N-terminus. HKD is the catalytic domain and hydrolyzes phosphatidylcholine into signal

69 molecule phosphatidic acid (PA) and soluble choline. Emphatically with PA as a key lipid

70 second messenger, PLD2 participates in many biological processes, such as cell proliferation,

71 membrane trafficking, cytoskeleton reorganization and cell migration (Bruntz et al., 2014). Both

$72 \mathrm{PX}$ and $\mathrm{PH}$ are regulatory domains with many novel functions revealed recently. The PX domain

73 acts as guanine nucleotide exchange factor (GEF) transforming the small GTPase, such as Rac2

74 or RhoA, from GDP-bound to active GTP-bound form, and thus stands for membrane ruffling

75 and stress fiber formation, importantly independent of the lipid activity (Ganesan et al., 2015;

76 Jeon et al., 2011; Mahankali et al., 2012; Ponting et al., 1996; Wishart et al., 2001). PLD also

77 interacts directly with cytoskeleton proteins, such as actin, tubulin and Colifin (Chae et al., 2005;

78 Han et al., 2007; Kusner et al., 2003; Lee et al., 2001). PLD1/2 depletion result in actin

79 cytoskeleton defects in macrophage cells (Ali et al., 2013). Not only the cytoskeleton

80 reorganization function is reported in the physiologic cell processes, many researches also 
81 mention PLD2 involvement in the metastasis of cancer. High level of PLD2 is found in 82 colorectal and breast cancers, implying its possible role in cancer invasion and metastasis 83 (Oshimoto et al., 2003).

84 As PLD2 plays a key role in cytoskeleton reorganization in many cells and oocyte maturation is a process propelled by a series cytoskeleton dynamic changes, what role of PLD2 in the meiotic division is of great interests for us to discover. In this study, we detected stable expression of PLD2 in mouse oocytes during meiotic maturation, and its requirement for the normal assembly and peripheral migration of meiotic spindle, independent on its catalytic activity in PA production.

\section{MATERIALS AND METHODS}

\section{Animal experiments ethics and feeding condition}

All the animal experiments were strictly conducted following the policies and instructions of the Care and Use of Animals in Research and Teaching and approved by the Animal Care and Use Committee of Capital Medical University with the approval No AEEI-2015-119. Mice C57BL/6male and BALB/C-female, together with their F1 off-springs were raised as the $12 \mathrm{~h}$ light /dark cycle in a proper humidity and temperature with enough food and water.

\section{Oocyte collection and culture}

Ovaries were harvested from 21-day-old female $\mathrm{F} 1$ mice which were euthanatized with $\mathrm{CO}_{2}$ at 44-48 $\mathrm{h}$ after intraperitoneal injection of $10 \mathrm{IU}$ pregnant mare serum gonadotropin (PMSG, Beijing XinHuiZeAo Science and Technology). Cumulus cell-oocyte complexes (COCs) were released from ovary follicles by puncturing the ovaries with 25-gauge needles, and further 
104

105

106

107

108

109

110

111

112

113

114

115

116

117

118

119

120

121

122

123

124

125

126

cultured in Minimal Essential Medium (MEM) with 10\% fetal bovine serum (FBS, Gibco) and 3 $\mathrm{mg} / \mathrm{ml}$ bovine serum albumin (BSA, Sigma) in an atmosphere with $5 \% \mathrm{CO}_{2}$ and proper humidity at $37^{\circ} \mathrm{C}$. At $0,2,4,8$ and $17 \mathrm{~h}$ of culture, corresponding to meiotic stages at germinal vesicle (GV), germinal vesicle breakdown (GVBD), pro-metaphase I (pro-MI), metaphase I (MI) and metaphase II (MII), respectively, the cumulus cells were deposed and oocytes were processed for different experiments. As for the drug treatment, denuded oocytes were used and departed into control and drug- MEM and IVM to different periods and ready for other procedures.

\section{Pharmacological inhibition of PLD2}

In order to analyze the potential function of PLD2 in mouse oocytes, three PLD2 inhibitors were used in this study, including PLD1/2 dual inhibitor 5-Fluoro-2-indolyl des-chlorohalopemide (FIPI) (sc-300694, Santa Cruz Biotechnology), and the specific PLD2 inhibitor, N-[2-[1-(3Fluorophenyl)-4-oxo-1, 3,-8-triazaspiro [4.5] dec-8-yl] ethyl]-2-naphthalenecarboxamide (NFOT) (4171, TOCRIS Bioscience), and 1-butanol (537993, Sigma). FIPI binds at S757 of PLD2, which is within the HKD2 catalytic site of the enzyme, rapidly blocking in vivo PA production with subnanomolar potency. NFOT is mixed-kinetics inhibitor, binding to PLD2 at two different sites, one being at S757/S648 in HKD domain, and another being an allosteric site that is a natural phosphoinositide biding pocket in PH domain and usually occupied by PIP2. NFOT affects both PA production and PIP2 binding to PLD2. And 1-butanol is another PLD inhibitor, blocking PLD production of PA, and widely employed to identify PLD/PA-driven processes.

All solid drugs were reconstructed to stock solution at $50 \mathrm{mM}$ in dimethyl sulfoxide (DMSO, Sigma), and further diluted in oocyte culture medium to final working concentration before use. For control, same amount of DMSO was added in the medium. The quantity of DMSO was not 
127 more than $0.1 \%(\mathrm{v} / \mathrm{v})$ in working solution. 1-butanol was resupplied every hour for the reduced 128 effect.

129

130 Cooling treatment

131 Oocytes at GV stage were pre-cultured for $8 \mathrm{~h}$ in maturation medium supplemented with DMSO 132 or NFOT, at which time, the majority oocytes were supposed to develop to MI stage. After 133 thoroughly washed, these oocytes were placed on ice and incubated in fresh M2 medium (M5910, 134 Sigma) for additional $20 \mathrm{~min}, 40 \mathrm{~min}$ and $60 \mathrm{~min}$, respectively, and then processed for analysis 135 of microtubules and PLD2 through immunofluorescence procedure.

\section{Immunofluorescence}

Oocytes were fixed in 1\% paraformaldehyde (PFA) in PEM buffer (100 mM Pipes, $1 \mathrm{mM} \mathrm{MgCl} 2$ and $1 \mathrm{mM}$ EGTA, $\mathrm{pH}$ 6.9) with $0.5 \%$ Triton $\mathrm{X}-100$ for $30 \mathrm{~min}$ at room temperature. After triple washing in phosphate-buffered saline (PBS) containing 0.2\% Triton X-100 (PBST), oocytes were blocked in PBST with 10\% normal goat serum, 1\% BSA and $0.3 \mathrm{M}$ glycine for $1 \mathrm{~h}$ at room temperature, and incubated in properly diluted primary antibodies, including rabbit anti-PLD2 (1:1000; HPA013397, Sigma), mouse anti-acetylated tubulin (1:3000; T7451, Sigma), mouse anti-PIP2 (1:500; sc-53412, Santa Cruz Biotechnology), mouse anti-phosphor Colifin (Ser3) (1:1000; GTX50199, GeneTex) and mouse anti-RhoA (1:500; sc-418, Santa Cruz Biotechnology), at $4^{\circ} \mathrm{C}$, overnight. After triple washing in PBST, each for $10 \mathrm{~min}$, oocytes were treated with goat anti-mouse Alexa-488 (1:500; Molecular Probes) or goat anti-rabbit Alexa-594 (1:500; Molecular Probes) in a light-proof box for $45 \mathrm{~min}$ at room temperature. After washed as described, oocytes were mounted on the glass slides with Vectashield mounting medium 
150 containing DAPI (H-1200, Vector Laboratories). All images were taken by DP-97 Olympus

151 microscope.

152 The actin staining was carried out following special procedure as described previously ( $\mathrm{Na} J$ $153 \&$ Zernicka-Goetz M, 2006). Oocytes were exposed to the acidic Tyrode's solution (T1788, 154 Sigma) for 2 min to remove the zona pellucida. After short recovery in culture medium, these 155 oocytes were fixed in the fix solution (4\% PFA, 0.15\% glutaraldehyde, $0.06 \%$ Triton X-100, 130 $156 \mathrm{mM} \mathrm{KCl}, 25 \mathrm{mM}$ HEPES and $3 \mathrm{mM} \mathrm{MgCl}_{2}, \mathrm{pH} 6.9$ ), for $30 \mathrm{~min}$ at room temperature, and then 157 permeabilized in $0.2 \%$ Triton X-100 in PEM buffer prior to additional 10 min incubation in fix 158 solution. After thoroughly washed, these cells were labeled with Alexa flour 555 - phalloidin (1:3000; 8953, Cell Signaling Technology). The samples were mounted and analyzed as 160 described above.

\section{Western blot}

50 oocytes in each sample were collected in Laemmli lysis buffer (161-0737, Bio-Rad) with protease inhibitor cocktail (P2714, Sigma) and stored at $-80^{\circ} \mathrm{C}$. Before use, the samples were degenerated at $100^{\circ} \mathrm{C}$ for $5 \mathrm{~min}$ and cooled down on ice. The proteins were separated by $10 \%$ SDS-PAGE and blotted to PVDF membrane (IPVH00010, Millipore), the membranes were then blocked in 5\% non-fat milk in Tris-buffered saline (TBS) containing $0.1 \%$ Tween-20 (TBST) for $1 \mathrm{~h}$ at room temperature, and then incubated overnight at $4^{\circ} \mathrm{C}$ in diluted primary antibodies. After washed three times, each for $15 \mathrm{~min}$, the membranes were further labeled with horseradish peroxidase-conjugated secondary antibodies (ZSGB-BIO) for $45 \mathrm{~min}$ at room temperature. After triple washes as described, the membranes were treated with enhanced chemiluminescence (ECL) system (P1010, Applygen Technologies Inc.). The semi-quantitative gray scale analysis of bands 
173

174

175

176

177

178

179

180

181

182

183

184

185

186

187

188

189

190

191

192

193

194

195

was processed using Image $\mathrm{J}$ software.

\section{Data statistics}

All experiments were performed at least 3 replicates and 30-50 oocytes were included in each group. The statistics and graphs were processed and produced by GraphPad Prism 5.01 (GraphPad Software, La Jolla,CA, USA). Data were given as the mean $\pm \mathrm{SEM}$ and $\mathrm{P}<0.05$ was considered significant.

\section{RESULTS}

\section{Stable expression of PLD2 and its relationship with spindle in oocyte meiosis}

The protein expression of PLD2 in mouse oocytes was firstly detected by western blot analysis. As showed in Figure 1, high level of PLD2 protein expression was revealed in mouse oocytes, importantly, the protein level was consistently stable from germinal vesicle (GV) stage to metaphase II (MII) (Fig. 1A), and further statistical analysis of band gray scale confirmed there was no significant difference in PLD2 level among different meiotic stages, as checked at GV, germinal vesicle breakdown (GVBD), metaphase I (MI) and MII stage, respectively (Fig. 1B).

Then the subcellular distribution pattern of PLD2 was explored with immunofluorescence approach. As showed in Figure 1, no special aggregation was observed in oocytes at GV stage (Fig. 1C, c), indicating PLD2 was evenly distributed in cytoplasm before the resumption of meiosis. Upon GVBD, as the chromatin was condensed into individual chromosomes (Fig. 1C, e), PLD2 began to aggregate around the condensing chromosomes, simultaneously with the newly formed microtubules (Fig. 1C, f-h). As cell cycle progressed to pro-metaphase I (pro-MI) and MI stage, microtubules were gradually organized into bi-polar spindle with all the 
196 chromosomes properly aligned on the equatorial plate, evidently, PLD2 sustained its co-

197 localization with microtubules throughout the whole process of spindle organizing (Fig. 1C, j-l,

198

199

200

201

202

203

204

205

206

207

208

209

210

211

212

213

214

215

216

217 218

n-p). The co-localization pattern lasted to the coming anaphase, during which PLD2 was overlapped with microtubules at polar area (Fig. 1C, r-t). When oocytes developed to MII stage, PLD2 was again distributed across the re-formed spindle and precisely co-localized with microtubules (Fig. 1C, u-x). These data strongly suggest possible involvement of PLD2 in meiotic spindle formation or positioning in mouse oocytes.

\section{PLD2 inhibition suppressed spindle migration to the cortex during oocyte meiosis}

We intended to explore the possible role of PLD2 in oocyte meiotic maturation by using specific inhibitor to PLD2. After GV oocytes were cultured for $17 \mathrm{~h}$ with NFOT at $20 \mu \mathrm{M}$, which was a defined proper concentration after series of experiments, nearly all the oocytes were arrested at MI stage but not matured to MII, further statistical analysis demonstrated that the number of oocytes with first polar body extrusion was significantly lower in NFOT group than that in control (Fig. 2B). Importantly, all the MI oocytes were installed with an enlarged spindle which was center-positioned (Fig. 2A).

We went back to check spindle structure and positioning at $8 \mathrm{~h}, 10 \mathrm{~h}$ and $12 \mathrm{~h}$ of NFOT incubation. As showed in Figure 2C, all the oocytes remained at MI stage in NFOT group (Fig. $\mathbf{2 C}$, a'-c'), no matter the incubation time extended up to $12 \mathrm{~h}$, and the spindle was enlarged and located closely to the central area (Fig. 2C, d'-f'). PLD2 was co-localized with microtubule on spindle and aggregated as big dots, distributed near to spindle poles (Fig. 2C, g': arrows) and randomly in cytoplastic area (Fig. 2C, g': arrowhead). In contrast, nearly in all control oocytes, the spindle exhibited normal size and was located in the sub-cortex position (Fig. 2C, d-e), some 
219 oocytes developed to MII stage as culture time increased to $12 \mathrm{~h}$ (Fig. 2C, c). To quantitatively 220 compare spindle changes after NFOT treatment, we measured the value of long axis length of 221 spindle (1), oocyte spherical radius (R) and the distance between spindle center and oocyte 222 spherical center $(\mathrm{d})$ in each oocyte. The ratio of "l" to " $\mathrm{R}$ " indicated the relative value of spindle

223

224

225

226

227

228

229

230

231

232

233

234

235

236

237

238

239

240

241

length and was denoted as " $L$ " $(L, L=1 / R)$. The ratio of " $d$ " to " $R$ " indicated the relative value of spindle migrating distance and was denoted as "D" (D, D = d/R) (Fig. 2 D). Statistical analysis demonstrated that the "L" value was significantly higher in NFOT-treated oocytes than that in control at $8 \mathrm{~h}, 10 \mathrm{~h}$ and $12 \mathrm{~h}$ incubation (Fig. 2E) $(\mathrm{P}<0.05)$, indicating the spindle was dramatically enlarged with PLD2 inhibition, at the same time, the " $\mathrm{D}$ " value was markedly decreased in NFOT group at all the time points (Fig. 2F) $(\mathrm{P}<0.001)$, implying spindle migrating toward the cortex was suppressed in oocytes when PLD2 was inhibited with NFOT.

As the enlarged spindle, we proposed that suppression of PLD2 may change the organizing pattern of spindle microtubules, and this proposal was proved by the classic cold-induced microtubule depolymerization experiment. When MI oocytes were incubated on ice for different periods with NFOT, we found that microtubules were more stable in NFOT-treated oocytes. As showed in Figure 3, microtubules were totally disassembled in control oocytes after $20 \mathrm{~min}$ incubation on ice (Fig. 3, a), simultaneously, PLD2 assembly was also disappeared (Fig. 3, e). However, in NFOT group, microtubules remained with PLD2 in polar area at 20 min of incubation (Fig. 3, b, f), even detectable at $40 \mathrm{~min}$ (Fig. 3, c, g), and totally depolymerized when checked at 60 min (Fig. 3, d, h). Microtubule dynamics was highly consistent with PLD2 signal during the whole process of cold treatment. These data indicate that PLD2 is associated with normal stability of microtubules, and PLD2 inhibition could promote microtubule stability, might contributing to the formation of large spindle. 


\section{Catalytic inhibition of PLD2 did not affect the cortex-towards spindle movement.}

244

245

246

247

248

249

250

251

252

253

254

255

256

257

258

259

260

261

262

263

264

PLD2 catalyzes the hydrolysis of phosphatidylcholine to produce PA and choline, and this function is dependent on PLD2 catalytic domain HKD. NFOT is an allosteric and catalytic inhibitor, targets two different sites in HKD and PH domain, suppressing both PA production and PIP2 binding to PLD2. FIPI is specific catalytic inhibitor to PLD and binds to HKD domain, inhibiting PA production (Monovich et al., 2007). The alcohol 1-butanol also blocks PLDcatalyzed PA generation (McDermott et al., 2004). In order to clarify whether PLD2 catalytic activity was involved in spindle migration, oocytes were treated with two catalytic inhibitors, FIPI and 1-butanol, in the same manner of NFOT. Beyond our expectation, we were astonished to find that FIPI nearly exerted no effect on spindle migration even in a cell-death dose (Fig. 4). Similarly, 0.1\% 1-butanol did not disrupt the spindle movement but indeed enlarged the size of spindle (Fig. 5). There data inferred that PLD2 HKD domains probably do not take part in the regulation of spindle cortex-towards migration.

\section{PLD2 suppression altered actin dynamics in mouse oocytes}

As the spindle disabled in movement under the condition of PLD2 inhibition with NFOT, we went back to assay if any changes occurred in actin network and relative several proteins, such as PIP2 and Colifin. With specific method of actin fixation, we found that actin was presented in a fibro-crossing manner throughout the cytoplasm in control oocytes, however, in the NFOT treatment group, actin distribution changed to forming many clusters quite big in size on the oocyte surface, with no obvious actin fiber crossover in cytoplasm (Fig. 6). It is plausible that PLD2 inhibition by NFOT resulted in great change in oocyte actin distribution, which led to the 
265 disable movement of spindle from accentor to the cortex.

266 Since PIP 2 and Colifin are tightly associated with actin polymerization and dynamics, we 267 continued to examine the cellular level of these proteins in NFOT-treated oocytes. By 268 immunofluorescence in normal MI oocytes, we found that both PIP2 and phosphorylated Colifin 269 (p-Colifin ${ }^{\mathrm{Ser} 3}$ ) were aggregated as bright foci at the poles of spindles (Fig. 7A, b; B, c: arrows) 270 and also randomly in cytoplasm area (Fig. 7A, b; B, c: arrowheads), just like the signal of 271 microtubule orgalizing center (MTOC), however, the foci signal of PIP2 definitely disappeared 272 in oocytes incubated with NFOT (Fig. 7A, f), similarly, p-Colifin ${ }^{\text {Ser3 }}$ signal was also significantly 273 weakened or totally disappeared in NFOT-treated oocytes (Fig. 7B, g). Further statistical 274 analysis confirmed that the number of oocytes with bright foci of PIP2 or p-Colifin ${ }^{\text {Ser3 }}$ was 275 significantly reduced after NFOT treatment (Fig. 7C, D) $(\mathrm{P}<0.001)$. In consistence, western blot 276 analysis indicated $\mathrm{p}-\mathrm{Colifin}{ }^{\mathrm{Ser} 3}$ protein level was reduced greatly while PLD2 level remained 277 stable (Fig. 7E), and this was supported by statistical data that the band gray intensity of p278 Cofilin ${ }^{\text {Ser3 }}$ was significantly decreased in NFOT-treated oocytes (Fig. 7F). The two weakened signals indirectly indicate that at least at the spindle poles the actin was more likely to be in a disassembly state under the condition of PLD2 inhibition, which may stand for the unmoved 281 spindles.

282 In addition, we found the small GTPase RhoA, which can bind with PLD2 and regulate actin 283 dynamics, was organized into a bi-polar structure with PLD2 localized mainly at the polar area in MI oocytes (Fig. 8). In NFOT-treated oocytes, RhoA sustained in "spindle-like" assembly, and also concentrated as additional dots in the cytoplasm, which were co-localized with the cytoplasmic PLD2 dots (Fig. 8A, f-h: arrows). Results of statistical analysis confirmed the proportion of oocytes with cytoplasmic RhoA dots was pronouncedly higher than that in control 
288

289

290

291

292

293

294

295

296

297

298

299

300

301

302

303

304

305

306

307

308

309

310

(Fig. 8B) $(\mathrm{P}<0.001)$. The changed distribution of RhoA must affect its function in regulation of actin polymerization and dynamics. NFOT-induced big dots in the cytoplasm are newly aggregated structure, may have some relationship with some transport-vesicles in the cytoplasm such as Golgi stacks and endosomes.

\section{DISCUSSION}

The present study shows that PLD2 is consistently expressed in mouse oocytes during meiotic maturation and co-localized with microtubules on spindle structure. PLD2 inhibition can affect microtubule stability and suppress spindle migration to the cortex, through regulating some actin-related factors, but independent of its catalytic activity.

We suppressed PLD2 activity by specific inhibitor NFOT and found that the meiotic progression was impeded as the enlarged spindle remained in the center, unable to approach the cortex. By using FIPI, which inhibited both PLD1/2 by inhibit the catalytic (HKDs) domains, we were surprised to find that spindle movement was not much impaired even in a lethal dose. Again, 1-butanol, which cuts off the production of PA, did not affect the peripheral migration of spindle. As NFOT has dual-inhibited sites on PLD2, one in the regulatory domain, and the other in the catalytic domain, so we suppose that PLD2 may take part in the spindle migration through a PA-independent way, in other words, not by its catalytic domain. Although many researchers have showed that the PA is a key lipid second messenger in many biological processes, however, we probably proved the rest roles of PLD2.

In oocytes with PLD2 inhibition, the spindle was obviously enlarged, suggesting normal PLD2 activity may be associated with mechanism responsible for spindle size maintenance. In mammalian oocytes, mitogen-activated protein kinase (MAPK) activity regulates microtubule 
311 organizing and spindle length (Sun QY et al., 1999). Its family member p38a MAPK cooperates

312 with kinesin molecule Eg5 to maintain the scale magnitude of spindle structure in oocytes, the

313 former works to hold the spindle magnitude while the latter functions just the opposite. The

314 depletion of p38 $\alpha$ MAPK could induce spindle elongation and meiotic arrest in mouse oocytes

315 (Ou XH et al., 2010), this phenotype is similar with that in PLD2-inhibited oocytes. Actually,

316 PLD2 has been proved to be involved in activating MAPK pathway (Rizzo et al., 1999). The

317 signal crossroad PLD2 - PA - diacylglycerol (DAG) mediates the activation of p38-MAPK

318 (Yang et al., 2010), and PLD2 also activates Ras-MAPK-1L-2 in T cells (Hamdi et al., 2008).

319 These evidences imply PLD2 and MAPK pathway may work together to regulate spindle

320 assembly in oocytes, whether spindle length maintenance is PLD2 downstream effect mediates

321 by MAPK needs further experiments.

322 It is well known that spindle relocation relies on actin network in mouse oocytes, we searched

323 to find proteins or mediators that not only regulate actin dynamics but also have potential

324 interaction with PLD2, especially the none-catalytic domain, conveying the effect onto the actin.

325 It has been indicated for years that PLD2 is a GEF for activating small GTPase RhoA (Gomez-

326 Cambronero J. 2011; Jeon H et al. 2011), and a previous study shows that RhoA can regulate

327 actin dynamics during porcine oocyte maturation (Zhang Y et al., 2014). In the present study, we

328 found that PLD2 could interact directly with RhoA in mouse oocytes, and in condition of PLD2

329 inhibition, both PLD2 and RhoA were aggregated into big dots in the cytoplasm, in addition to

330 co-localization with microtubule on spindle, suggesting RhoA as a potential candidate that

331 mediates the PLD2 effect on actin network. PIP2 is well-known as an essential lipid messenger

332 at the plasma membrane, where its localized generation regulates endocytosis, exocytosis, actin

333 cytoskeleton dynamics, focal adhesion assembly, ion channels and transporters (Tan et al., 2015). 
334 Recent evidences demonstrate that reduced PIP2 synthesis suppresses actin polymerization and

335 motility, while increasing PIP2 synthesis enhances these activities (Breitbart H \& Finkelstein M

336

337

338

339

340

341

342

343

344

345

346

347

348

349

350

351

352

353

354

355

356

2015). PIP2 binds specifically to the PH domain of PLD isoforms with high affinity and independent of the substrate phosphatidylcholine, implying a key role of the PH domain in PLD function (Hodgkin et al., 2000; Sciorra et al., 2002), and coordination between PLD2 and PIP2 in regulating actin polymerization and function. NFOT inhibits PLD2 activity by blocking PIP2 binding with PLD2. As we found in this study, the polar location of PIP2 was disrupted in NFOT-treated oocytes, suggesting the local state of actin polymerization at spindle polar area must be altered, and logically accounts for the delayed migration of spindle. Colifin is known to sever actin filaments by creating more positive ends on filament fragments, this actindepolymerizing protein can be phosphorylated at Ser3 and inactivated by LIMK1, thus an increasing p-Cofilin ${ }^{\text {Ser3 }}$ level can promote actin polymerization (Pfender et al., 2011; Sun et al., 2011). It is newly revealed that Cofilin is a PIP2-binding protein in somatic cells (Sengelaub $C A$ et al., 2016), consistent with our finding that both PIP2 and p-Colifin ${ }^{\mathrm{Ser} 3}$ were localized at the polar area of spindle in mouse oocytes, and interestedly, this polar recruitment was destroyed by PLD2 inhibition. Collectively, the information may suggest PIP2, p-Cofilin ${ }^{\text {Ser3 }}$ and PLD2 are components of super complex at spindle poles, and individually, PLD2 serves as a scaffolding plate, PIP2 interacts with PLD2 and mediates p-Cofilin ${ }^{\text {Ser3 }}$ binding to the complex, thus these molecules work together to regulate actin polymerization and spindle migration. PLD2 dysfunction would affect the complex formation and inhibit spindle towards-cortex migration.

In summary, the data presented here suggest that PLD2 is involved in spindle formation and its migration to the cortex in mouse oocytes during meiotic progression, particularly this function is independent of PLD2 catalytic activity in phosphatidylcholine hydroxylation. 


\section{ACKNOWLEDGMENTS}

359

360

361

362

363

364

365

366

367

368

369

370

371

372

373

374

375

376

377

378

379

The authors thank Dr. Qian Wang, Dr. Juan Du, Dr. Yuanjing Liang and Ms. Jing Weng for their technical help and critical reading of the manuscript.

\section{REFERENCES}

Ali WH, Chen Q, Delgiorno KE, Su W, Hall JC, Hongu T, Tian H, Kanaho Y, Di Paolo G, Crawford HC, Frohman MA. 2013. Deficiencies of the lipid-signaling enzymes phospholipase D1 and D2 alter cytoskeletal organization, macrophage phagocytosis, and cytokine-stimulated neutrophil recruitment. PLoS One 8:e55325 DOI:

10.1371/journal.pone.0055325

Almonacid M, Paoletti A. 2010. Mechanisms controlling division-plane positioning. Seminars in Cell \& Developmental Biology 21: 874-880～DOI: 10.1016/j.semcdb.2010.08.006

Breitbart H, Finkelstein M. 2015. Regulation of Sperm Capacitation and the Acrosome Reaction by PIP 2 and Actin Modulation. Asian Journal of Andrology 17(4):597-600. doi: 10.4103/1008-682X.154305

Bruntz RC, Lindsley CW, Brown HA. 2014. Phospholipase D signaling pathways and phosphatidic acid as therapeutic targets in cancer. Pharmacological Review 66:1033-1079 DOI: $10.1124 /$ pr.114.009217

Chae YC, Kim KL, Ha SH, Kim J, Suh PG, Ryu SH. 2010. Protein kinase Cdelta-mediated phosphorylation of phospholipase D controls integrin-mediated cell spreading. Molecular and Cellular Biology 30:5086-5098ＤOI: 10.1128/MCB.00443-10

Chae YC, Lee S, Lee HY, Heo K, Kim JH, Kim JH, Suh PG, Ryu SH. 2005. Inhibition of 
380

381

382

383

384

385

386

387

388

389

390

391

392

393

394

395

396

397

398

399

400

401

402

muscarinic receptor-linked phospholipase $\mathrm{D}$ activation by association with tubulin. The Journal of Biological Chemistry 280: 3723-3730ＤOI: 10.1074/jbc.M406987200

Chaigne A, Campillo C, Gov NS, Voituriez R, Azoury J, Umana-Diaz C, Almonacid M, Queguiner I, Nassoy P, Sykes C, Verlhac MH, Terret ME. 2013. A soft cortex is essential for asymmetric spindle positioning in mouse oocytes. Nature cell biology 15: 958-966 DOI: $10.1038 /$ ncb2799

Chaigne A, Verlhac MH, Terret ME. 2012. Spindle positioning in mammalian oocytes. Experimental Cell Research 318: 1442-1447 DOI: 10.1016/j.yexcr.2012.02.019

Dumont J, Million K, Sunderland K, Rassinier P, Lim H, Leader B, Verlhac MH. 2007. Formin-2 is required for spindle migration and for the late steps of cytokinesis in mouse oocytes. Developmental Biology 301:254-265ＤOI: 10.1016/j.ydbio.2006.08.044

Ganesan R, Mahankali M, Alter G, Gomez-Cambronero J. 2015. Two sites of action for PLD2 inhibitors: The enzyme catalytic center and an allosteric, phosphoinositide biding pocket. Biochimica et Biophysica Acta 1851: 261-272ＤOI: 10.1016/j.bbalip.2014.12.007

Gomez-Cambronero J. 2011. The exquisite regulation of PLD2 by a wealth of interacting proteins: S6K, Grb2, Sos, WASp and Rac2 (and a surprise discovery: PLD2 is a GEF). Cellular Signalling 23:1885-1895ＤOI: 10.1016/j.cellsig.2011.06.017

Gonczy P. 2002. Mechanisms of spindle positioning: focus on flies and worms. Trends in Cell Biology 12: 332-339.

Hamdi SM, Cariven C, Coronas S, Malet N, Chap H, Perret B, Salles JP, Record M. 2008. Potential role of phospholipase D2 in increasing interleukin-2 production by T-lymphocytes through activation of mitogen-activated protein kinases ERK1/ERK2. Biochimica et Biophysica Acta 1781: 263-269 DOI: 10.1016/j.bbalip.2008.03.005 
Han L, Stope MB, de Jesús ML, Oude Weernink PA, Urban M, Wieland T, Rosskopf D, Mizuno K, Jakobs KH, Schmidt M. 2007. Direct stimulation of receptor-controlled phospholipase D1 by phospho-Colifin. The EMBO journal 26: 4189-4202 DOI: 10.1038/sj.emboj.7601852

Hodgkin MN, Masson, MR, Powner D, Saqib KM, Ponting CP, Wakelam MJ. 2000. Phospholipase D regulation and localisation is dependent upon a phosphatidylinositol 4,5biphosphate-specific PH domain. Current Biology 10: 43-46.

Jeon H, Kwak D, Noh J, Lee MN, Lee CS, Suh PG, Ryu SH. 2011. Phospholipase D2 induces stress fiber formation through mediating nucleotide exchange for RhoA. Cell Signalling 23: 1320-1326 DOI: 10.1016/j.cellsig.2011.03.014

Kusner DJ, Barton JA, Qin C, Wang X, Iyer SS. 2003. Evolutionary conservation of physical and functional interactions between phospholipase D and actin. Archives of Biochemistry and Biophysics 412:231-241 DOI: 10.1016/s0003-9861(03)00052-3

Leader B, Lim H, Carabatsos MJ, Harrington A, Ecsedy J, Pellman D, Maas R, Leder P. 2002. Formin-2, polyploidy, hypofertility and positioning of the meiotic spindle in mouse oocytes. Nature Cell Biology 4:921-928～DOI: 10.1038/ncb880

Lee S, Park JB, Kim JH, Kim Y, Kim JH, Shin KJ, Lee JS, Ha SH, Suh PG, Ryu SH. 2001. Actin directly interacts with phospholipase D, inhibiting its activity. The Journal of Biological Chemistry 276:28252-28260 DOI: 10.1074/jbc.M008521200

Li H, Guo F, Rubinstein B, Li R. 2008. Actin-driven chromosomal motility leads to symmetry breaking in mammalian meiotic oocytes. Nature Cell Biology 10:1301-1308 DOI: $10.1038 / \mathrm{ncb} 1788$

Mahankali M, Henkels KM, Alter G, Gomez-Cambronero J. 2012. Identification of the 
426

427

428

429

430

431

432

433

434

435

436

437

438

439

440

441

442

443

444

445

446

447

448

catalytic site of phospholipase D2 (PLD2) newly described guanine nucleotide exchange factor activity. The Journal of biological chemistry 287:41417-41431 DOI:

10.1074/jbc.M112.383596

Maro B, Verlhac MH. 2002. Polar body formation: new rules for asymmetric divisions. Nature Cell Biology 4:E281-283ＤOI: 10.1038/ncb1202-e281

Na J, Zernicka-Goetz M. 2006. Asymmetric positioning and organization of the meiotic spindle of mouse oocytes requires CDC42 function. Current Biology 16:1249-54 DOI: 10.1016/j.cub.2006.05.023

Oshimoto H, Okamura S, Yoshida M, Mor M. 2003. Increased activity and expression of phospholipase D2 in human colorectal cancer. Oncology Research 14: 31-37.

Ou XH, Li S, Xu BZ, Wang ZB, Quan S, Li M, Zhang QH, Ouyang YC, Schatten H, Xing FQ, Sun QY. 2010. p38alpha MAPK is a MTOC-associated protein regulating spindle assembly, spindle length and accurate chromosome segregation during mouse oocyte meiotic maturation. Cell Cycle 9:4130-4143 DOI:10.4161/cc.9.20.13389

Pfender S, Kuznetsov V, Pleiser S, Kerkhoff E, Schuh M. 2011. Spire-type actin nucleators cooperate with Formin-2 to drive asymmetric oocyte division. Current Biology 21: 955-960 DOI: $10.1016 /$ j.cub.2011.04.029

Ponting CP. 1996. Novel domains in NADPH oxidase subunits, sorting nexins, and PtdIns 3kinases: binding partners of SH3 domains. Protein Science 5:2353-2357 DOI:

$$
10.1002 / \text { pro.5560051122 }
$$

Rizzo MA, Shome K, Vasudevan C, Stolz DB, Sung TC, Frohman MA, Watkins SC, Romero G. 1999. Phospholipase D and its product, phosphatidic acid, mediate agonistdependent raf-1 translocation to the plasma membrane and the activation of the mitogen- 
activated protein kinase pathway. Journal of Biological Chemistry 274:1131-1139

450

451

452

453

454

455

456

457

458

459

460

461

462

463

464

465

466

467

468

469

470

471

\section{Sathananthan AH, Selvaraj K, Girijashankar ML, Ganesh V, Selvaraj P, Trounson AO.} 2006. From oogonia to mature oocytes: inactivation of the maternal centrosome in humans. Microscopy research and technique 69: 396-407 DOI: 10.1002/jemt.20299

Schuh M. 2011. An actin-dependent mechanism for long-range vesicle transport. Nature Cell Biology 13: 1431-1436 DOI: $10.1038 / \mathrm{ncb} 2353$

Schuh M, Ellenberg J. 2008. A new model for asymmetric spindle positioning in mouse oocytes. Current Biology 18: 1986-1992ＤOI: 10.1016/j.cub.2008.11.022

Sciorra VA, Rudge SA, Wang J, McLaughlin S, Engebrecht J, Morris AJ. 2002. Dual role for phosphoinositides in regulation of yeast and mammalian phospholipase D enzymes. The Journal of Cell Biology 159: 1039-1049ＤOI: 10.1083/jcb.200205056

Sengelaub CA, Navrazhina K, Ross JB, Halberg N, Tavazoie SF. 2016. PTPRN2 and PLC $\beta 1$ promote metastatic breast cancer cell migration through $\mathrm{I}(4,5) \mathrm{P} 2$-dependent actin remodeling. The EMBO Journal 35(1):62-76. doi: 10.15252/embj.201591973.

Sun QY, Breitbart H, Schatten H. 1999. Role of the MAPK cascade in mammalian germ cells. Reproduction Fertility and Development 11:443-450

Sun SC, Wang ZB, Xu YN, Lee SE, Cui XS, Kim NH. 2011. Arp2/3 complex regulates asymmetric division and cytokinesis in mouse oocytes. PLoS One 6:e18392 DOI: 10.1371/journal.pone.0018392

Tan XJ, Thapa N, Choi SY, Anderson RA. 2015. Emerging roles of PtdIns(4,5)P2 - beyond the plasma membrane. Journal of Cell Science 128: 4047-4056 doi: 10.1242/jcs.175208

Zhang Y, Duan X, Cao R, Liu HL, Cui XS, Kim NH, Rui R, Sun SC. 2014. Small GTPase RhoA regulates cytoskeleton dynamics during porcine oocyte maturation and early embryo 
472 development. Cell Cycle 13(21):3390-403. doi: 10.4161/15384101.

473 von Dassow G. 2009. Concurrent cues for cytokinetic furrow induction in animal cells. Trends

474 in Cell Biology 19:165-173 DOI: 10.1016/j.tcb.2009.01.008

475 Wishart MJ, Taylor GS, Dixon JE. 2001. Phoxy lipids: revealing PX domains as

476 phosphoinositide binding modules. Cell 105: 817-820.

477

478

479

480

481

482

483

484

485

486

Yang L, Seifert A, Wu D, Wang X, Rankovic V, Schroder H, Brandenburg LO, Hollt V, Koch T. 2010. Role of phospholipase D2/phosphatidic acid signal transduction in microand delta-opioid receptor endocytosis. Molecular Pharmacology 78:105-113 DOI:10.1124/mol.109.063107

Yi K, Rubinstein B, Li R. 2013. Symmetry breaking and polarity establishment during mouse oocyte maturation. Philosophical transactions of the Royal Society of London. Series B, Biological sciences 368:20130002ＤOI: 10.1098/rstb.2013.0002

Yi K, Rubinstein B, Unruh JR, Guo F, Slaughter BD, Li R. 2013. Sequential actin-based pushing forces drive meiosis I chromosome migration and symmetry breaking in oocytes. The Journal of Cell Biology 200:567-576 DOI: 10.1083/jcb.201211068 


\section{Figure 1 (on next page)}

The protein expression of PLD2 and its relationship with spindle in mouse oocytes during meiotic maturation

A. Western blot analysis detected stable expression of PLD2 at GV, GVBD, MI and MIl stage, respectively. The experiments were repeated triply. B. Statistical analysis confirmed no significant difference in PLD2 expression among germinal vesicle (GV), germinal vesicle breakdown (GVBD), metaphase I (MI) and metaphase II (MII) stage (P>0.05). C. PLD2 was colocalized with spindle during meiotic division. At GV stage, no particular aggregation of PLD2 was detected throughout the cytoplasm and nuclear (a-c). After GVBD, PLD2 emerged as filamentous assembly and was co-localized with newly formed microtubules around the condensed chromosomes (e-h). From pro-MI to MI, PLD2 was co-localized with the spindle structure (i-p), and during anaphase I (AI) to telophase I (Tel I), it was mainly distributed at spindle poles and absent from midbody area (q-t). At MII stage, PLD2 was again co-localized with microtubules on the re-formed meiotic spindle and first polar body (1 $\left.{ }^{\text {st }} \mathrm{PB}\right)(\mathrm{u}-\mathrm{x}$ : arrow). DNA was visualized in blue, microtubules were in green and PLD2 was in red. Scale bar $=20$ $\mu \mathrm{M}$. 
A

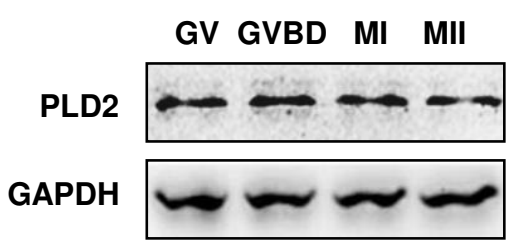

C

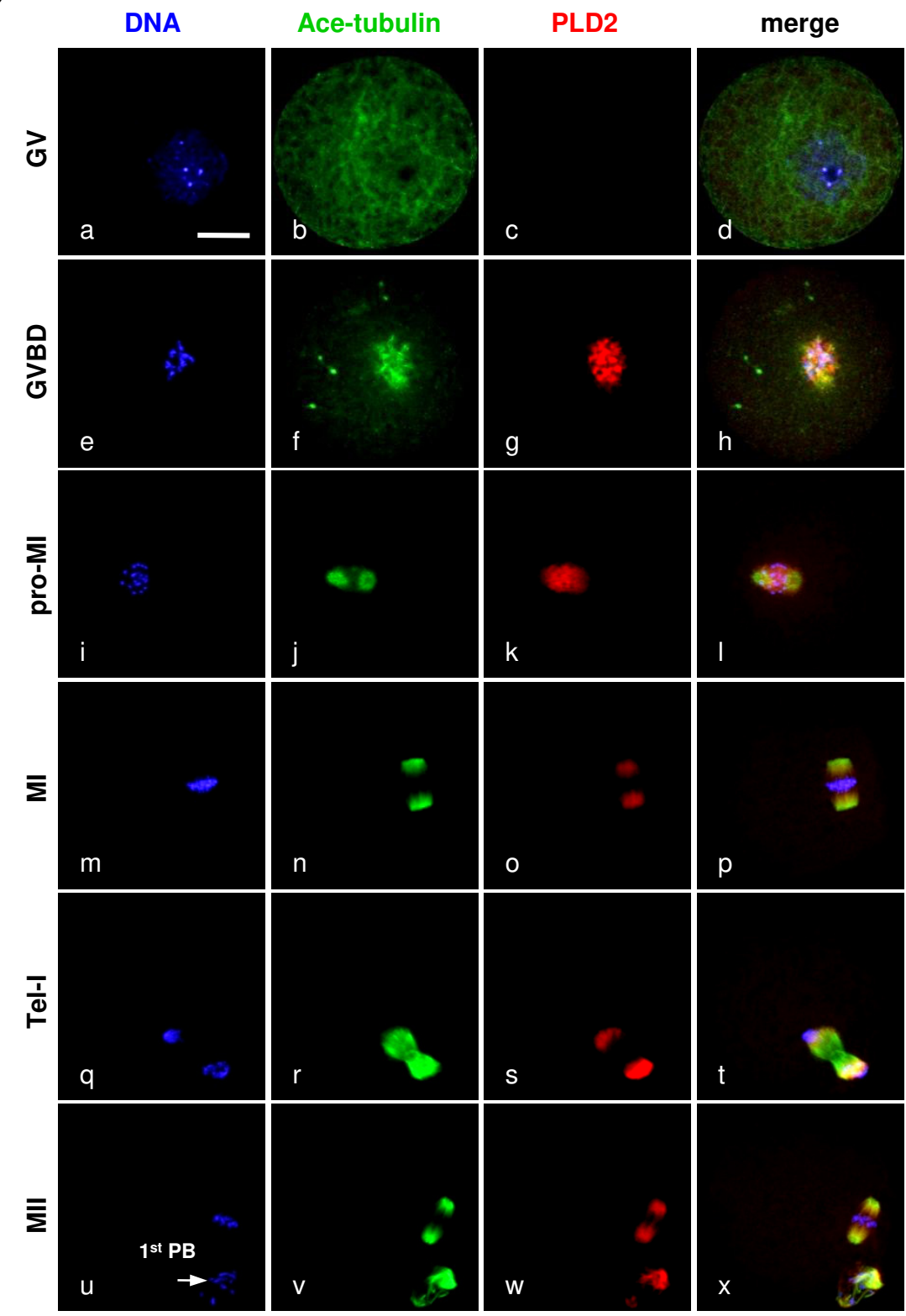

$1^{\text {st }}$ PB: first polar body
B

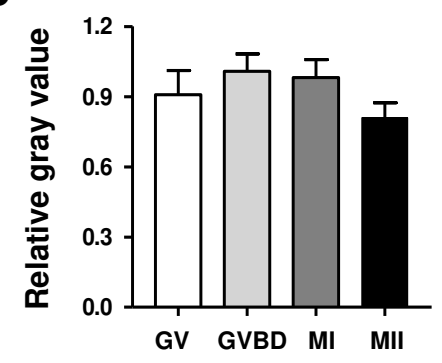




\section{Figure 2 (on next page)}

PLD2 inhibition resulted in center-positioned spindle and arrested meiotic progression

GV oocytes were in vitro cultured for $8 \mathrm{~h}, 10 \mathrm{~h}, 12 \mathrm{~h}$ and $17 \mathrm{~h}$, respectively, in maturation medium with $20 \mathrm{mM}$ NFOT, and then collected for immunofluorescence analysis. Oocytes incubated with only DMSO was used as control. The experiment was repeated at least three times with more than 30 oocytes analyzed in each group every time. DNA was labeled in blue, microtubules were in green and PLD2 was in red. Scale bar $=20 \mu \mathrm{M}$. A. Representative images showed center-positioned spindle with enlarged size and meiotic arrest at MI after 17 $\mathrm{h}$ incubation in NFOT (e-h), PLD2 was labeled on spindle and also exhibited as big dots located in the polar area and cytoplasm (g: arrow). Control oocytes developed to MII stage with normal spindle size and PLD2 distribudtion (a-d, arrow: first polar body, 1 st PB). B. Result of statistical analysis demonstrated the number of MII oocytes, manifested with extruded 1 st PB, was significantly lower in NFOT group after $17 \mathrm{~h}$ incubation $(\mathrm{P}<0.001)$. C. Representative images showed spindle status and meiotic progression in oocytes when checked at $8 \mathrm{~h}, 10 \mathrm{~h}$ and $12 \mathrm{~h}\left(\mathrm{~d}^{\prime}-\mathrm{f}^{\prime}\right)$, in NFOT-treated oocytes, meiotic progressed was arrested at MI stage with enlarged spindle nearly at the center area, PLD2 was labeled on spindle and also as large dots in area near to spindle poles ( $g$ ': arrows) and across the cytoplasm ( $g$ ': arrowhead). In oocytes incubated with DMSO alone, the spindle was assembled in normal size and positioned in the cortex (d, e), PLD2 was co-localized with microtubules on spindle $(\mathrm{g}-\mathrm{i})$, some oocytes were progressed to MII stage when checked at $12 \mathrm{~h}$ (c, f, i, l: arrow: 1 st PB). D. Computational method for measurement of spindle size and migration distance. Red dot indicates the spherical center of oocytes and blue dot indicates the center of spindle, "l" indicates the length of spindle long axis, "R" indicates the spherical radius of oocytes, and " $d$ " indicates the distance from spindle center to spindle center; " $L$ " is the ratio value between "I" and " $R$ " $(L=l / R)$, representing the relative length of spindle structure, " $D$ " is the ratio value between " $d$ " and " $R$ ", indicating the relative distance of

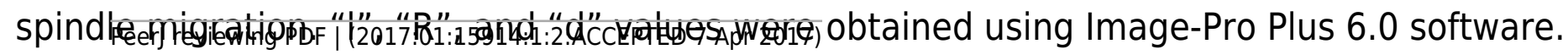


E. The result of statistical analysis indicated the spindle size ("L" value) was significantly bigger in NFOT-treated oocytes than that in control when analyzed at $8 \mathrm{~h}, 10 \mathrm{~h}$ and $12 \mathrm{~h}$ of drug incubation $(P<0.05)$. F. Statistical data indicated the distance of spindle migrating to the cortex ("D" value) was significantly smaller in NFOT-treated oocytes than that in control when analyzed at $8 \mathrm{~h}, 10 \mathrm{~h}$ and $12 \mathrm{~h}$ of drug incubation $(\mathrm{P}<0.001)$. 


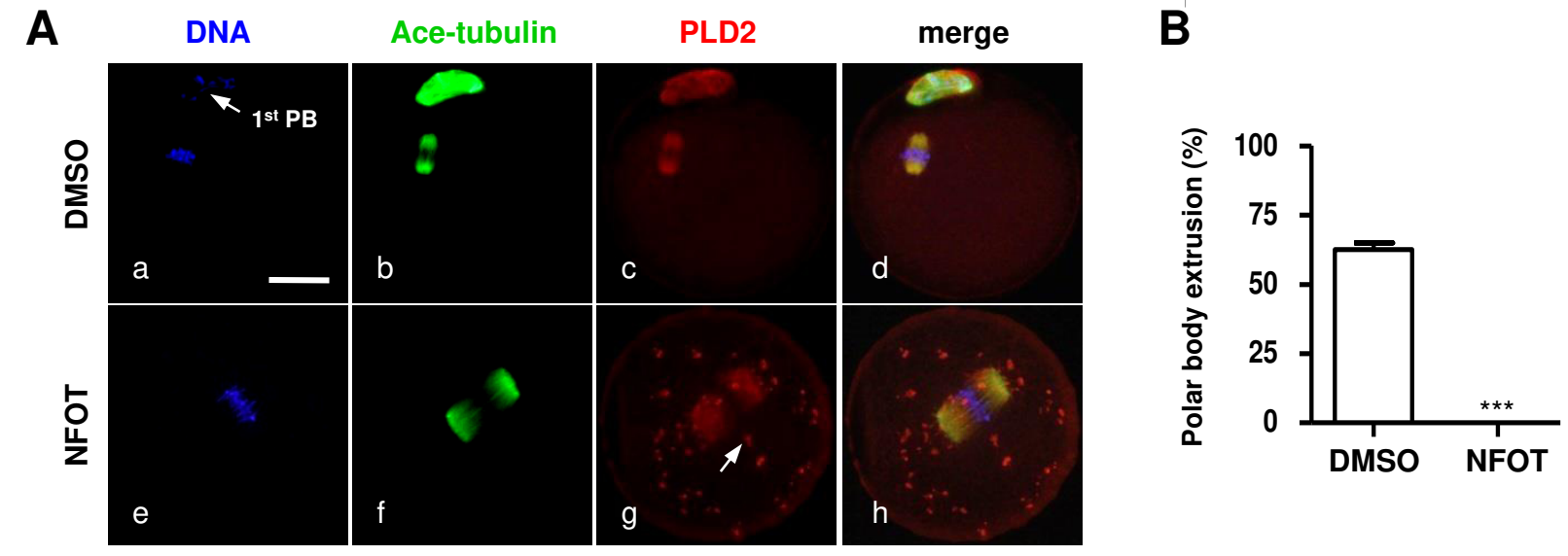

B

Manuscript to be reviewed
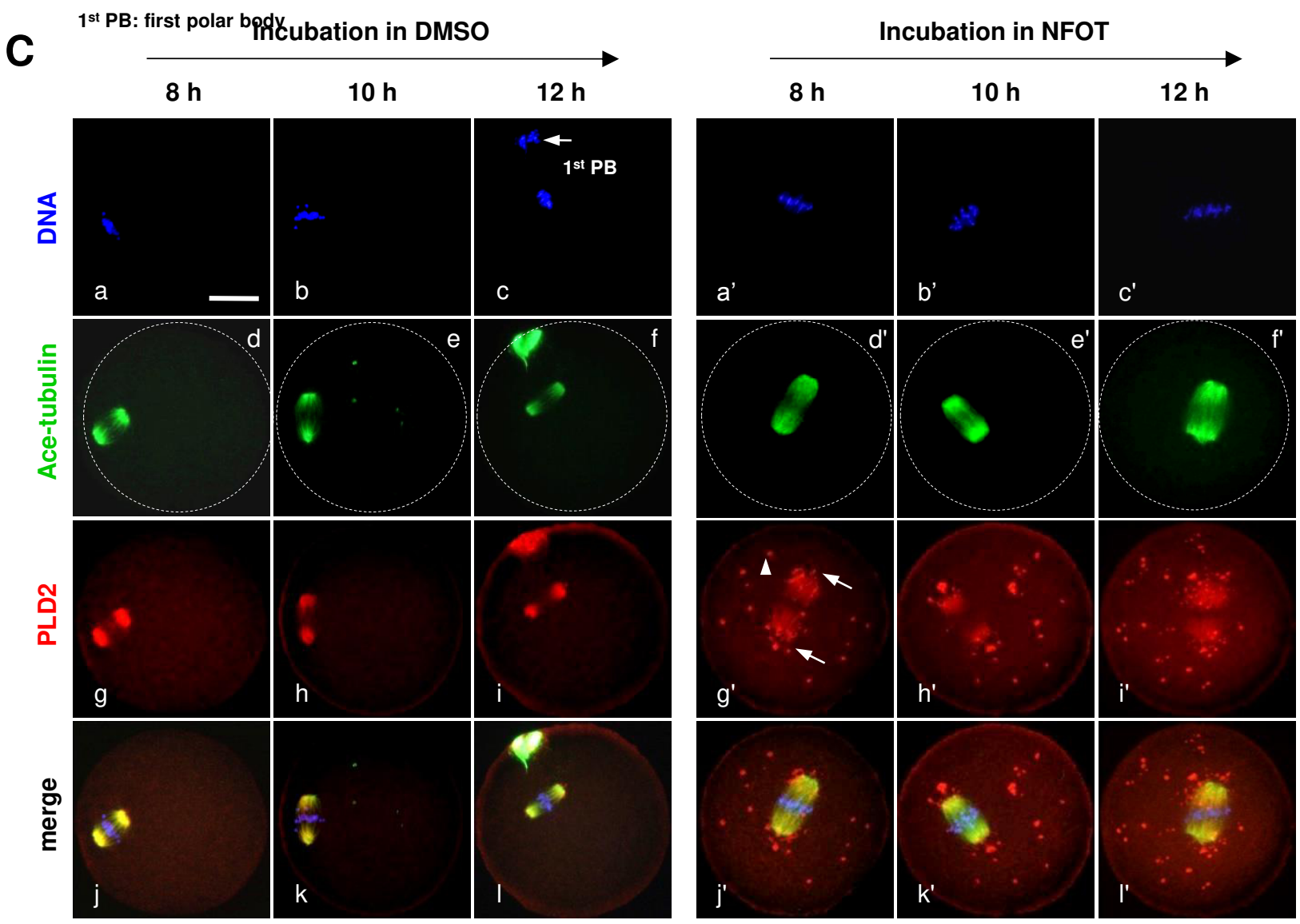

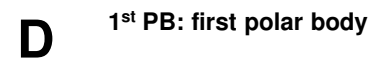

E

$\mathbf{F}$

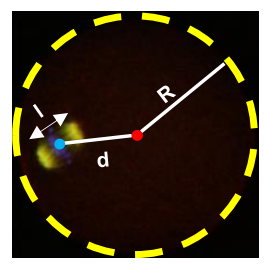

$\mathbf{L}=\mathbf{I} / \mathbf{R}$

$D=d / R$

I: spindle length

$R$ : radius of oocyte

d: distance between centers of oocyte

and spindle

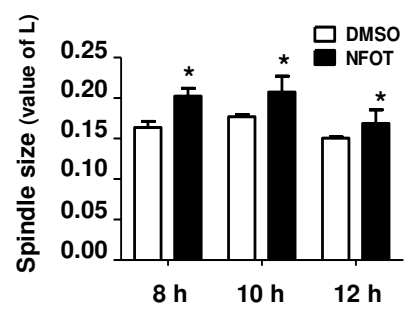

PeerJ reviewing PDF | (2017:01:15914:1:2:ACCEPTED 7 Apr 2017) 


\section{Figure 3 (on next page)}

The spindle microtubules were stabilized in oocytes with PLD2 inhibition with NFOT

GV oocytes were incubated for $8 \mathrm{~h}$ with DMSO or NFOT, and then collected for additional incubation in fresh M2 medium on ice for $20 \mathrm{~min}, 40 \mathrm{~min}$ and $60 \mathrm{~min}$, respectively, and followed by immunofluorescence procedure. The experiment was repeated triply with more than 30 oocytes analyzed in each group every time. Microtubules were labeled in green and PLD2 in red. Scale bar $=20 \mu \mathrm{M}$. At $20 \mathrm{~min}$ of cooling treatment, the microtubules were totally disassembled together with PLD2 in control oocytes, however, both microtubules and PLD2 remained in NFOT-treated oocytes at this time, even sustained at $40 \mathrm{~min}$, and completely vanished at $60 \mathrm{~min}$. 


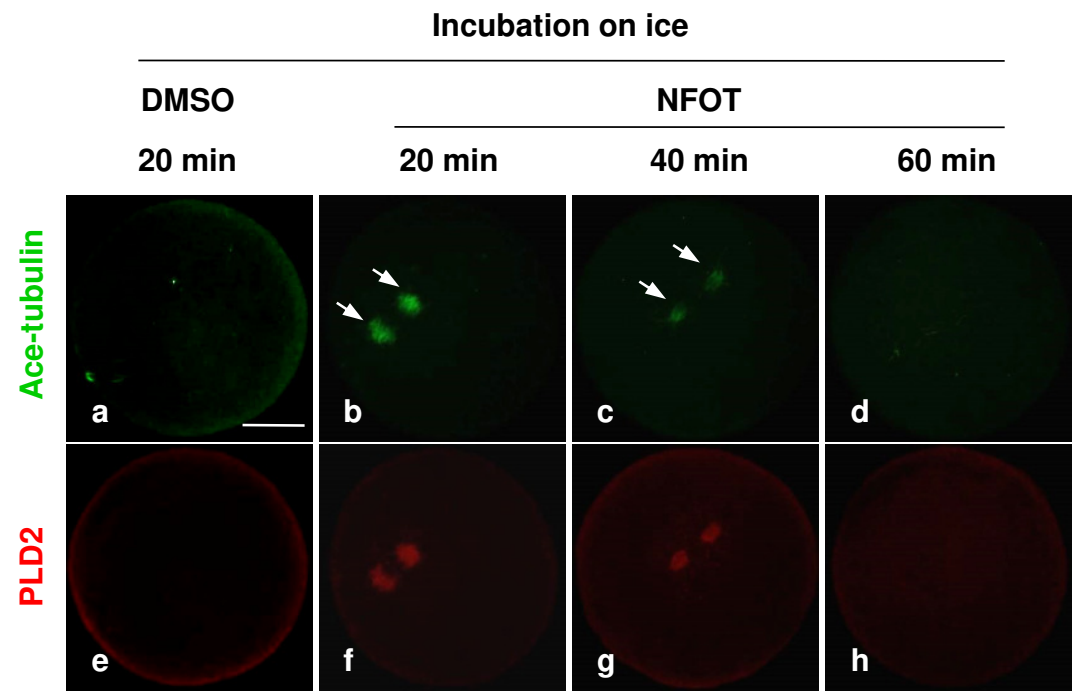




\section{Figure 4 (on next page)}

PLD catalytic inhibitor FIPlexerted no effect on spindle migration

GV oocytes were cultured for $17 \mathrm{~h}$ in maturation medium with DMSO, $12.5 \mu \mathrm{M}$ FIPI, $25 \mu \mathrm{M}$ FIPI and $37.5 \mu \mathrm{M} \mathrm{FIPI}$, respectively, and then fixed for immunofluorescence analysis. The experiment was repeated at least three times. DNA was labeled in blue, microtubules in green and PLD2 in red. Scale bar $=20 \mu \mathrm{M}$. A. Representative images illustrated meotic progression and spindle state after drug incubation. The majority oocytes progressed to MII stage in DMSO, $12.5 \mu \mathrm{M}$ FIPI and $25 \mu \mathrm{M}$ FIPI group, manifested with the extruded 1 st PB (a, e: arrow), the MI oocytes in these groups contained typical bi-polar spindles, which were organized in normal size and positioned in the cortex area (j). Nearly $27 \%$ oocytes were dead in $37.5 \mu \mathrm{M}$ FIPI group, but the rest MI oocytes were equipped with spindles in normal size and position (n-p). B. Statistical data indicated there was no significant difference in the number of $\mathrm{Ml}$ oocytes containing spindle in normal size and cortical position among all the treatment groups. 
A

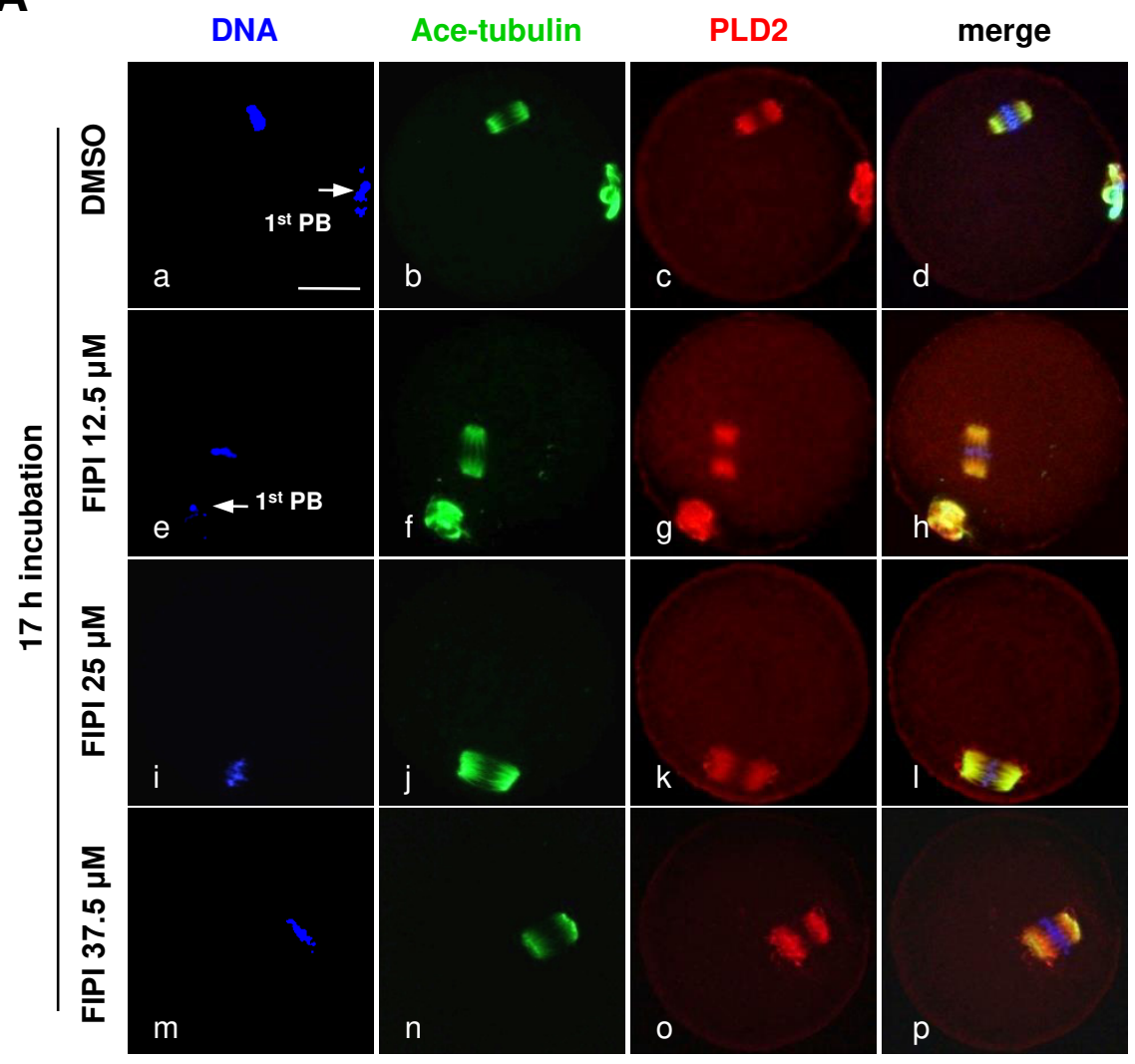

$1^{\text {st }}$ PB: first polar body

B

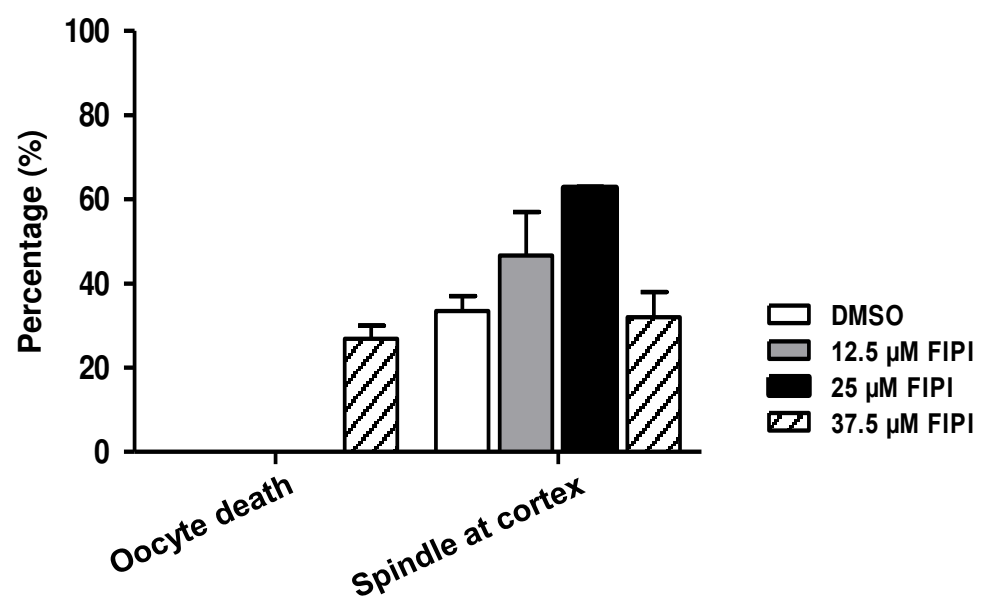




\section{Figure 5 (on next page)}

1-butanol did not affect spindle cortex migration

Oocytes at GV stage were cultured for $8 \mathrm{~h}$ in maturation medium containing $0.1 \%$ 1-butanol, and then fixed for immunofluorescence analysis. Three replicates were included in this experiment, and at least 30 oocytes were analyzed in each group every time. DNA was stained in blue, microtubules in green and PLD2 in red. Scale bar $=20 \mu \mathrm{M}$. A. Typical images illustrated the spindle was assembled in large size but positioned in the cortex after 1butanol incubation. B. Statistical data showed that the spindle was significantly enlarged in 1-butanol group than in control $(P<0.001)$. C. Results of statistical analysis confirmed 1brutanol had no effect on spindle migration toward the cortex. 

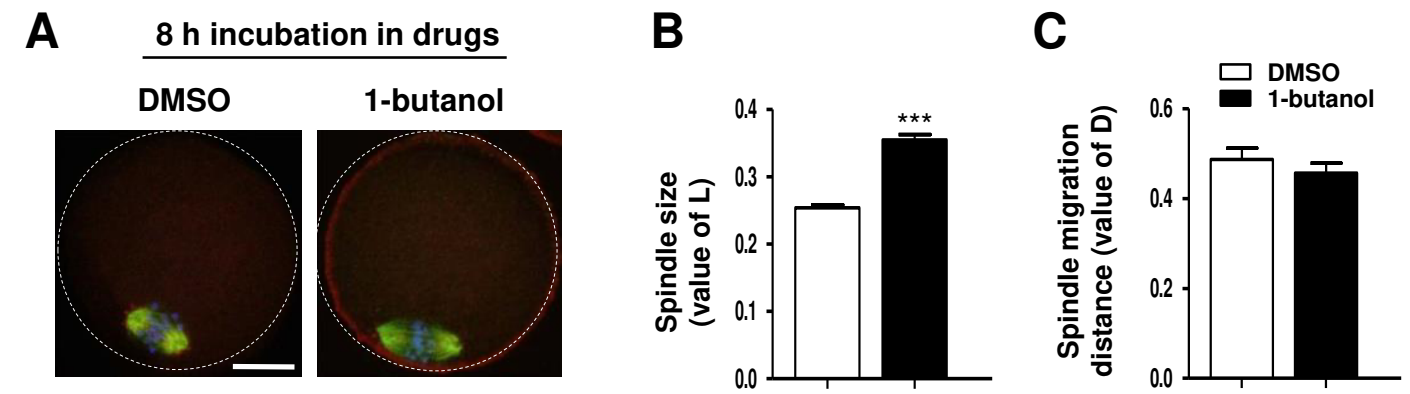

DNA Ace-tubulin PLD2 


\section{Figure 6}

Cytoplasmic distribution of actin was changed in NFOT-treated oocytes

Oocytes were labeled with Alexa flour 555 - phalloidin and DAPI after $8 \mathrm{~h}$ incubation with DMSO or NFOT. The experiment was repeated three times. DNA was visualized in blue and actin in red. Scale bar $=20 \mu \mathrm{M}$. A. In DMSO group, the actin was assembled as quite thick fibers in the cytoplasm, with pretty high concentration surrounding the spindle area (arrows in left image), while in the NFOT group, fibers were taken place by the big clusters (arrows in right image). B. Statistical analysis confirmed that the proportion of oocytes with actin clusters in cytoplasm was significantly higher in NFOT group $(\mathrm{P}<0.001)$.
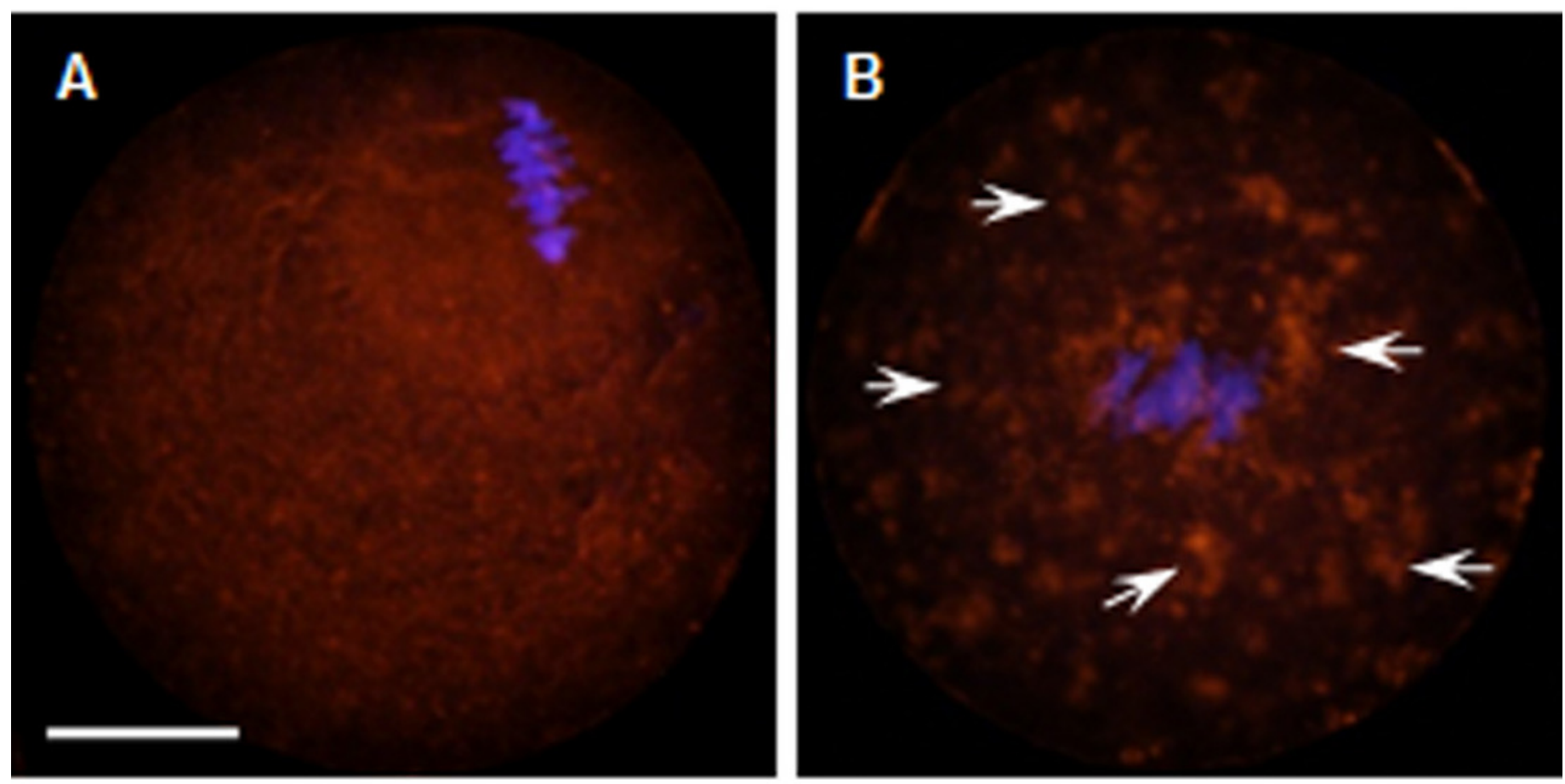

\section{Actin (phalloidin) DNA (DAPI)}




\section{Figure 7 (on next page)}

PLD2 inhibition destroyed polar recruitment of PIP2 and p-Cofilin ${ }^{\text {Ser3 }}$.

GV oocytes were cultured for $8 \mathrm{~h}$ in maturation medium with $20 \mathrm{mM}$ NFOT, and then fixed for analysis of PIP2 and p-Cofilin ${ }^{\text {Ser3 }}$. The experiment was repleated at least three times, and 100150 oocytes were included in each group every time. DNA was stained in blue, microtubules and PIP2 in green, and PLD2 and p-Cofilin ${ }^{\text {Ser3 }}$ in red. Scale bar $=20 \mu \mathrm{M}$. A.

Immunofluorescence showed PIP2 was concentrated on spindle polar area (b: arrows) and distributed as bright foci in the cytoplasmic area (b: arrowheads) in control oocytes, in contrast, PIP2 localization on spindle poles and distribution in cytoplasm was dramatically reduced or completely disappeared in NFOT-treated oocytes (f). B. Typical images illustrated that $\mathrm{p}$-Cofilin ${ }^{\mathrm{Ser} 3}$ was assembled as bright foci, which were localized on the poles of spindle (c: arrows) and also randomly distributed in the cytoplasmic area (c: arrowheads) in control oocytes, however, p-Cofilin ${ }^{\text {Ser3 }}$ aggregation on spindle poles and in cytoplasm was depleted in NFOT-treated oocytes $(\mathrm{g})$. C. Statistical data showed that the number of oocytes with bright PIP2 signal was significantly reduced in NFOT-treated oocytes than in control $(P<0.001)$. D. Statistical analysis confirmed that the proportion of oocytes with bright $p$-Cofilin ${ }^{\text {Ser3 }}$ was markedly decreased in NFOT group than in control $(P<0.001)$. E. Western blot analysis showed the protein expression of $p$-Cofilin ${ }^{\text {Ser3 }}$ was reduced in NFOT-treated oocytes, meanwhile, PLD2 expression was not affected. GAPDH expression was also detected and employed as loading control. D. Statistical analysis of blot band gray indicated p-Cofilin ${ }^{\text {Ser3 }}$ protein level was significantly lower in NFOT group than in control $(P<0.01)$. 
A

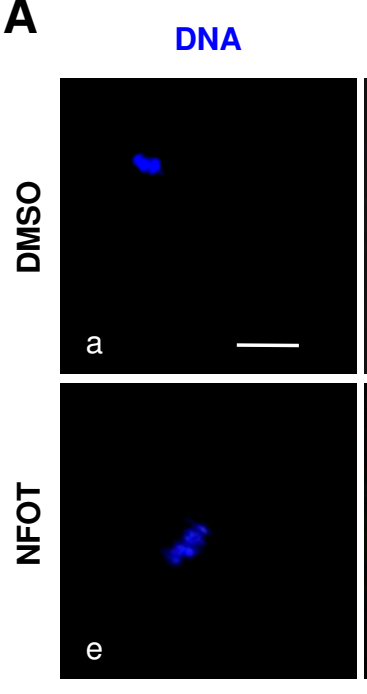

B

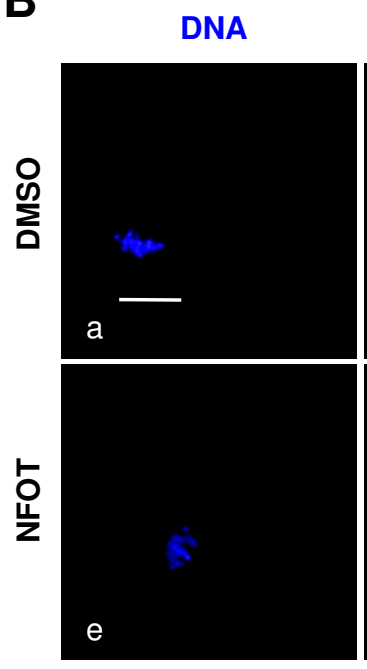

PIP2
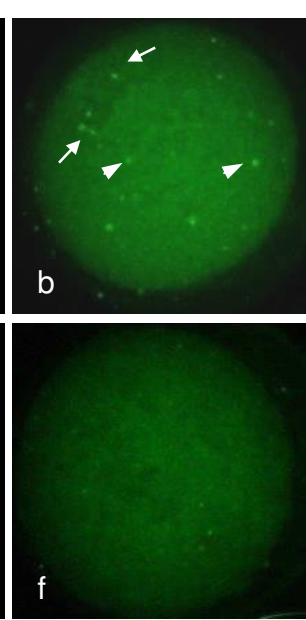

g

Ace-tubulin

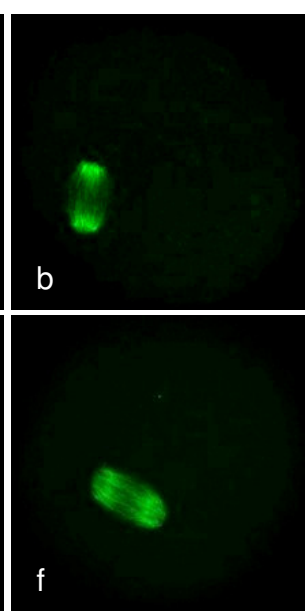

PLD2
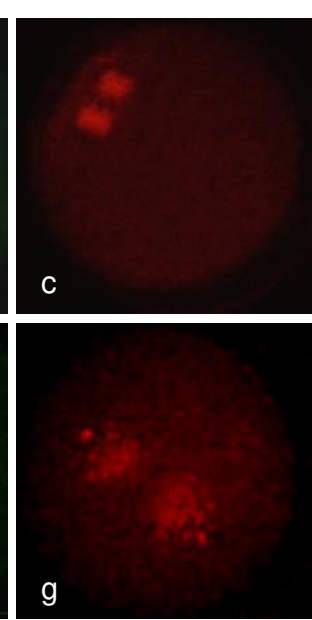

h

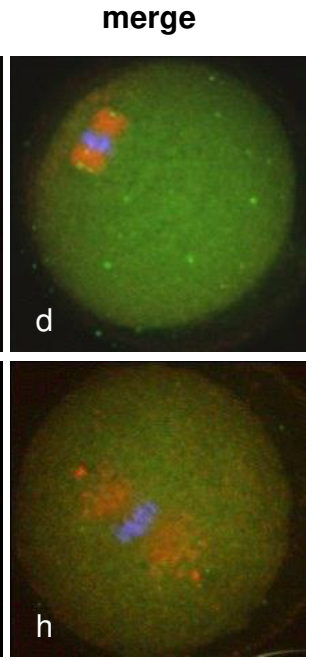

p-Cofilin

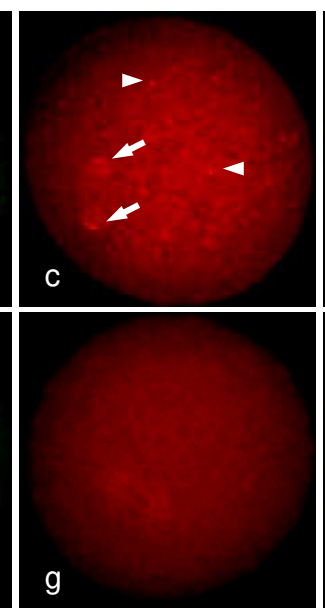

merge

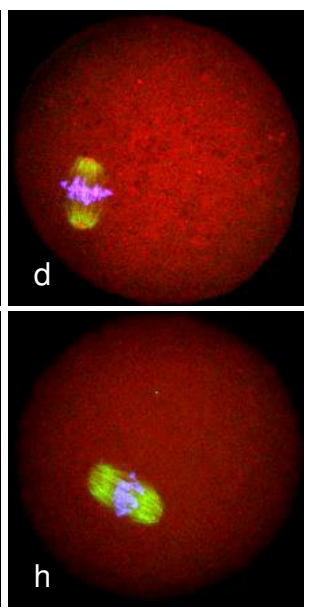

C
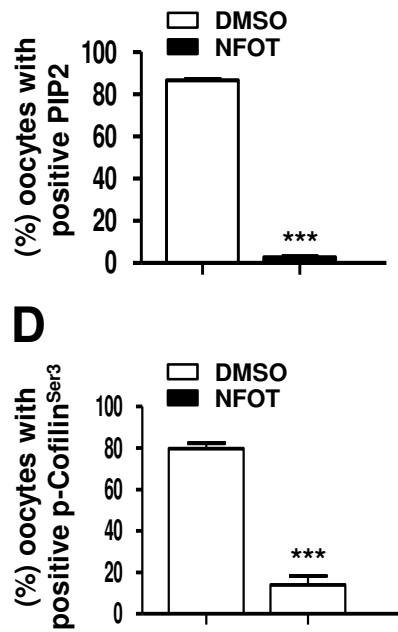

E

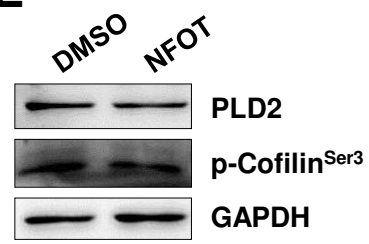

$\mathbf{F}$

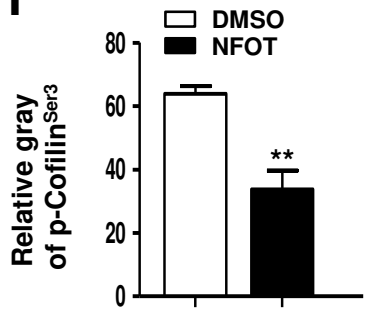




\section{Figure 8 (on next page)}

NFOT treatment changed RhoA cytoplasm distribution

Oocytes at GV stage were incubated for $8 \mathrm{~h}$ with or without NFOT, and then fixed for immunostaining of PLD2 and RhoA. This experiment was repeated three times, with 50 oocytes included in each group every time. DNA was labeled in blue, RhoA in green and PLD2 in red. Scale bar $=20 \mu \mathrm{M}$. A. Immunofluorescence showed co-localization of RhoA with PLD2 was only detected in the spindle area in control cells (b-c), however in NFOT-treated oocytes, RhoA was co-localized with PLD2 in spindle area, but also aggregated as foci and overlapped with PLD2 dots in cytoplasm (f-h: arrows). B. Statisticak analysis confirmed the proportion of oocytes with cytoplasmic RhoA dots was significantly higher in NFOT group $(P<0.001)$. 


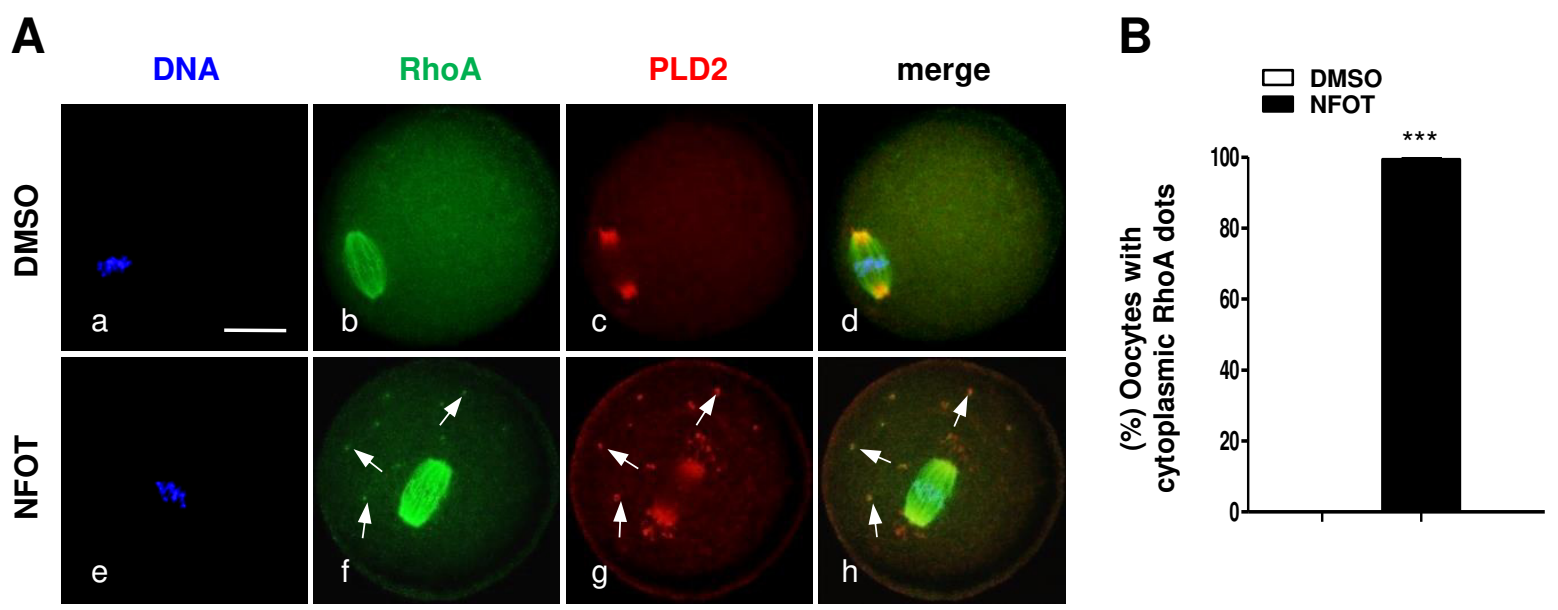

